\title{
LA TERMINOLOGÍA HOY: REPLANTEAMIENTO O DIVERSIFICACIÓN
}

\author{
María Teresa Cabré, Judit Freixa, Mercè Lorente, Carles Tebé
}

\begin{abstract}
RESUMO: Cet article présente d'abord le panorama actuel de la terminologie du point de vue théorique, qui se caractérise aujourd'hui par une grande diversité d'approches comme résultat de l'apparition de nouvelles nécessités au cours des dernières années, qui ont abouti à des usages terminologiques aussi très diverses. Comme réponse à cette nouvelle situation, les auteurs présentent une esquisse d'une proposition pour une nouvelle conception de la terminologie, qui essai d'intégrer des apports du côté linguistique, cognitive et sociale; cette nouvelle approche théorique présente aussi des conséquences importantes sur le plan méthodologique et pratique.
\end{abstract}

PALAVRAS-CHAVE: teoria terminológica; diversidade terminológica; poliedricidade dos termos; termo e contexto; variação terminológica, aplicações.

La terminología ha cambiado mucho en los últimos diez años. Este cambio se ha producido tanto en la práctica terminológica como en la teoría que subyace en ella. Lo cierto es que cada vez es mayor el número de especialistas en terminología que manifiestan abiertamente la insatisfacción que produce seguir sin matices los principios de la teoría general clásica, y han puesto en relive que entre la teoría establecida y las aplicaciones que se están llevando a cabo existen desajustes importantes, y ello por diferentes motivos.

Para explicarnos la importancia y el sentido de este giro en el enfoque de la terminología no podemos olvidar el panorama general y los cambios que se han producido en las materias que la configuran como una interdisciplina: la lingüística, la filosofía y la comunicación. Ni tampoco podemos prescindir del desarrollo de la inteligencia artificial y la informática aplicadas. Y menos aún de los cambios generales de tipo socioeconónico y sociopolítico que se han producido. Sin todos estos factores resultaría imposible explicarse las razones de los cambios, o más apropiadamente, de la flexibilización y la apertura, que se han producido en la teoría y la práctica terminológicas.

Cierto es que ha habido movimientos en la linguística reciente y que al lado del paradigma chomskiano dominante se han desarrollado modelos alternativos o complementarios sobre la base de la pragmática, la lingüística del texto y el funcionalismo de base sociológica. Paralelamente se ha profundizado en las aproximaciones de base cognitiva y se ha generalizado el interés por el conocimiento. El uso de la informática en los trabajos de reconocimiento, recopilación, clasificación y edición de terminología ha permitido abordar la terminografía con nuevos recursos y han conducido a modificaciones importantes en el proceso de trabajo. Estos factores, entre otros, han impulsado la necesidad de adaptar la teoría terminológica y su metodología de trabajo a las características de los nuevos aportes y a la vista de la diversidad de nuevas aplicaciones en atención a la gran disparidad de necesidades sociales entorno a la información y la comunicación.

En este artículo nos proponemos presentar a modo de difusión algunas líneas de un nuevo modelo de aproximación a la terminología, que pretende ser complementario a la propuesta clásica desarrollada en la Teoría General de la Terminologia (TGT) de Wüster. Esta nueva propuesta se nutre de aportes dispersos que algunos especialistas en terminología, de formación linguística, filosófica o traductológica han hecho a la teoría wüsteriana. Cabe citar en este sentido A. Rey 1979, y más recientemente los trabajos de Gambier 1991, Gaudin 1993, Sager 1990,

María Teresa Cabré, Judit Freixa, Mercè Lorente, Carles Tebé são professores da Facultat de Traducción e membros do Grupo IULATERM del Institut Universitari de Lingüística Aplicada (IULA) de la Universitad Pompeu Fabra, Barcelona.

Organon, Porto Alegre, nº 26, 1998 
Temermann 1997, Slodzian 1994, y Cabré, 1992, 1995 y 1999. Todos ellos han sugerido puntos de crítica a la TGT ante la imposibilidad no únicamente de explicar determinados fenómenos sobre la comunicación especializada y sus unidades más representativas, los términos, sino incluso de describir dichas unidades de forma que se pueda dar cuenta de su complejidad representativa y funcional.

La Socioterminología, inspirada en la sociolingüística y la teoría del análisis del discurso político francés (MARCELLESI y GUESPIN, 1986) abrió la primera brecha de crítica a la teoría clásica, pero no ha desarrollado hasta el momento una propuesta que permita sustentar una teoría nueva de la terminología.

En recientes trabajos de Cabré (1999) se establecen algunos pilares para una aproximación teórica renovada sobre el tema. Este artículo intenta recoger dichos puntos a modo de síntesis y comentario, a fin de darles una mayor difusión entre los especialistas y conseguir así un foro amplio de opiniones sobre la propuesta.

\section{LAS RAZONES DE LOS CAMBIOS}

Partimos pues de la base que el cambio hacia un nuevo enfoque terminológico más amplio tiene su marco, su punto de partida y también su justificación en otros cambios que se han producido a niveles más generales. A continuación vamos a hacer un breve repaso a estas evoluciones, concretamente en el campo de la lingüística y de la sociedad para ver como pueden haber provocado nuevos planteamientos y necesidades en el plano terminológico.

\section{Cambios en la lingüística}

Cabe destacar, por un lado, el crecimiento que ha experimentado la lingüística hacia el estudio del uso de la lengua. Efectivamente, a partir de los años sesenta el interés por el estudio de la estructura de la lengua se ha visto complementado por un claro incremento del interés por la descripción de su diversidad de usos por parte de los distintos hablantes, dando lugar a la aparición de la gramática de la variación. El interés por el análisis del texto ha jugado un papel preponderante en este cambio (CIAPUSCIO, 1998).

No se trata solamente de la aparición de nuevos modelos teóricos, que los ha habido, sino de un interés por un enfoque más comunicativo desde los distintos modelos. El lenguaje visto como vehículo de comunicación y socialización, de organización del pensamiento, de transmisión de creencias y de identificación, ha dado lugar a orientaciones de la lingüística inicialmente conocidos de forma genérica como estilística, que engloban visiones como la etnografía de la comunicación, el analisis del discurso y la pragmática. Todo ello, en el panorama de aparición de disciplinas, actualmente más o menos autónomas respecto de la lingüística, como són la sociolingüística y la psicolingüística.

La terminología, vista como disciplina lingüística, no ha quedado al margen de estas nuevas concepciones, basadas esencialmente en la variación de usos y usuarios y en la diversidad de funciones y situaciones comunicativas, y han adquirido gran importancia todos aquellos aspectos que pueden favorecer una explicación más satisfactoria de la comunicación especializada.

Aún en el plano lingüístico, la terminología se ha visto afectada por un aumento del intercambio entre lenguas. Así, de una situación de comunicación plurilingüe entre unas pocas lenguas de países cultural y tecnológicamente próximos — situación en la que fue diseñada la TGT—, hemos pasado a una situación de intercambio mucho más diversificado, entre muchas más lenguas, de familias alejadas y de estructura distinta.Y al lado de la tendencia a usar un número muy reducido de lenguas — que en muchas ocasiones llega al monolingüismo- para las comunicaciones internacionales, convive la tendencia aparentemente opuesta de defender las distintas lenguas nacionales con el fin de capacitarlas para cualquier tipo de comunicación, ya sea internacional o no, especializada o no.

\section{Cambios en la sociedad}

Han sido diversas las transformaciones que han vivido las sociedades, sobretodo a nivel socioeconómico, que han afectado directamente las necesidades terminológicas. La globalización del mercado, que provoca más intercambios, entre más lenguas y a niveles de especialización temática muy distintos, es tal

Organon, Porto Alegre, $\mathrm{n}^{\circ} 26,1998$ 
vez el más destacable de todos ellos, ya que ha dado lugar a un importante crecimiento geográfico y social de la ciencia y de la técnica, lo que ha provocado el acceso del público general a áreas muy especializadas y se han multiplicado los tipos de comunicaciones especializadas según el nivel de formación del público.

Por las dos razones que acabamos de ver, se ha producido una explosión de la técnica y la información, destacable por el crecimiento exponencial del conocimiento — en períodos cada vez más pequeños se producen avances tecnológicos cada vez más importantes- y por el carácter también exponencial de la información que se desprende de este conocimiento. El acceso a la información es cada vez más fácil —y para un público cada vez mayor-, más cómodo y más barato.

La relación de estos hechos con la terminología resulta evidente y puede resumirse en la necesidad de denominar una cantidad ingente de nuevos conceptos y de diversificar su transmisión para usos muy distintos.

\section{LA TGT Y LAS NUEVAS NECESIDADES}

Ante los cambios operados en la sociedad y en la lingüística en los últimos decenios, la TGT, que como corpus teórico nace al amparo de las ideas filosóficas del Círculo de Viena de los años treinta, presenta una visión de la realidad y de las lenguas excesivamente idealizada, que no es capaz de responder adecuadamente a los fenómenos de diversidad que acabamos de mencionar.

Las insuficiencias de la TGT se ponen de manifiesto especialmente en los puntos siguientes:

- Logicismo: el método de análisis lógico de la realidad es la forma 'científica' de describir el mundo. Este aspecto se pone de relieve, por ejemplo, en la descripción de los tipos de relaciones entre los conceptos, que aparecen dominadas por un modelo de organización del conocimiento de carácter jerárquico y binario. Los tipos de relaciones que escapan a este modelo están tan solo apuntados y no se hallan bien descritos.

- Universalismo: el método de análisis del conocimiento especializado tiene pretensión de validez universal, como manifiestan por ejemplo las normas sobre principios y métodos internacionales aprobadas por ISO, y por lo tanto este análisis parece aplicarse por igual a contextos geográficos, realidades socioeconómicas, culturales y lingüísticas completamente distintas.

- Estatismo: en efecto, aunque la TGT reconoce — siempre en letra pequeña- que los conceptos evolucionan, su modelo de representación del conocimiento tiene un carácter marcadamente estático, fruto de su propósito de adoptar una perspectiva de estudio estrictamente sincrónica, y no es capaz de integrar en su análisis de la realidad ningún elemento que de cuenta del carácter dinámico, evolutivo, del conocimiento especializado y de sus denominaciones.

- Reduccionismo: el ámbito original de aplicación de los trabajos terminológicos de la TGT, tanto en Centroeuropa como en la antigua Unión Soviética, era la técnica en general, y la mecánica y la ingeniería en particular. Al intentar extender este modelo de representación de la realidad a otras disciplinas (como las ciencias aplicadas, sociales y humanas), o bien a otros ámbitos especializados por criterios pragmáticos (como las profesiones, o sectores de ocio) tiene lugar una reducción sensible de la capacidad de descripción del modelo, que no puede dar cuenta de realidades tan dispares.

- Idealismo: como consecuencia de los puntos anteriores, la terminología parece querer reflejar un mundo idealizado, en el que los conceptos son entes preexistentes a las lenguas, creados por consenso en un laboratorio, y que etiquetan inequívocamente mediante los términos realidades con valor supralingüístico y supracultural.

Ya en los años noventa, todos estos supuestos, que podían agruparse bajo la característica común de una visión excesivamente idealizada de la realidad, han entrado en crisis, y han sido discutidos desde una triple prespectiva, que presentamos a continuación.

\section{PRINCIPALES PUNTOS DE DISCUSIÓN}

La complejidad de los lenguajes de especialidad, y por ende de sus sistemas terminológicos reales, se aprecia fácilmente si tenemos en cuenta la diversidad y dinamismo de las temáticas de trabajo y de situaciones comunicativas. La necesidad de referirnos, desde la disciplina terminológica, a esa complejidad, para

Organon, Porto Alegre, nº 26, 1998 
describirla, representarla y diseñar y realizar aplicaciones adecuadas en cada caso conlleva una revisión de algunos de sus postulados teóricos y metodológicos.

Aportaciones de este tipo provienen de la triple perspectiva con la que podemos estudiar los objetos terminológicos: la perspectiva social, la perspectiva cognitiva y la perspectiva lingüística. Desde la primera, en síntesis, se reclama una mayor adecuación de los fundamentos a las metodologías de trabajo para dar una respuesta mejor a las necesidades comunicativas de los profesionales y de los usuarios en general, que son tan cambiantes como lo es la sociedad. Desde la ciencia cognitiva, las principales críticas se orientan hacia la naturaleza misma del conocimiento especializado, y desde la lingüística, el uso de un modelo que abarque la competencia y la actuación. Así, la terminología, vista como una disciplina lingüística, debe dar respuesta a la descripción de los códigos, a la descripción de los actos comunicativos especializados reales, a la explicación del funcionamiento de la terminología dentro del lenguaje natural y a la elaboración de aplicaciones terminológicas diversas que cubran necesidades comunicativas también diversas.

Desde este posición, consideramos que los principales puntos de discusión que debemos abordar en la revisión teórica y metodológica de la disciplina están en relación con los siguientes ejes:

a) eje teórico de los fundamentos de la materia

b) eje metodológico del proceso de trabajo terminológico

c) eje estratégico de la construcción de aplicaciones

En síntesis, una visión lingüística de la terminología requiere que, tanto desde el punto de vista teórico como desde el metodológico, se contemple la variación lingüística en toda su complejidad, se sume la condición de adecuación de los términos y se recojan los aspectos psicolingüísticos implicados (compartidos con la perspectiva cognitiva) y los elementos sociolingüísticos relacionados (compartidos con la perspectiva social). Pero además es necesario que todos los objetos terminológicos (conocimiento especializado, textos especializados y unidades terminológicas) sean descritos en todos sus niveles de representación lingüística: semántico, fonológico, morfológico, sintáctico y pragmático.

\section{VARIOS ELEMENTOS A MODO DE ESBOZO DE PROPUESTA}

A partir de las propuestas de Cabré (1999), proponemos que debemos avanzar hacia un nuevo paradigma terminológico que, en el plano teórico, pueda integrar, entre otros, los elementos siguientes:

- La poliedricidad del término, ya que la unidad terminológica ofrece una triple perspectiva de estudio — social, lingüística y cognitiva-; estas tres perspectivas son complementarias.

- La pertenencia de los términos al sistema lingüístico; los términos no son unidades de un sistema artificial y autosuficiente como las nomenclaturas científico-técnicas; las reglas generales que gobiernan el funcionamiento del léxico son las mismas que gobiernan los términos. En este sentido los términos y las palabras se solapan.

- La variación como fenómeno normal, inherente al lenguaje; por ello, la variación debería estar adecuadamente contemplada y descrita en terminología, no eliminada ni reducida a priori a fin de favorecer una comunicación especializada precisa y unívoca, sin ambigüedades.

- La especialización de los conceptos por criterios temáticos o pragmáticos, sin entender que tengan un régimen ni un funcionamiento diferenciado respecto de los significados de la lengua general (que, obviamente, también pueden presentar especialización en distintos grados);

- El trasfondo sociocultural y lingüístico de una comunidad como factor que influye en los conceptos, así como los canales comunicativos por donde circulan y se difunden.

En síntesis, el modelo teórico que integre estas propuestas debería ser capaz de analizar las unidades terminológicas como unidades semántico-formales vinculadas a una lengua natural y caracterizadas por un sesgo cultural, y dar cuenta de sus características integrándolas en un modelo lingüístico (gramatical y pragmático).

\section{CONSECUENCIAS METODOLÓGICAS}

La aplicación real de éstos nuevos aspectos de la teoría terminológica a la metodología tiene

Organon, Porto Alegre, nº 26, 1998 
consecuencias a distintos niveles y, sin duda alguna, las más importantes tienen que ver con los principios terminográficos. Destacamos concretamente aspectos relacionados con la orientación onomasiológica, la representación de los sistemas conceptuales, la definición y la biunivocidad.

La orientación onomasiológica, única para el trabajo terminológico de metodología clásica se considera ahora preponderante pero no exclusivo ya que el uso de la informática en el trabajo terminológico para tareas de reconocimiento nos ha llevado a detectar el término a partir de su forma preponderantemente. Nos alejamos, además, de la visión estrictamente universalista del concepto y aceptamos la existencia de sesgos culturales cuando entendemos el concepto ligado a las distintas maneras de entender, representar y comunicar el conocimiento de cada sociedad.

Por otro lado, la representación de los sistemas conceptuales abandona su rigidez para dar cabida a tipos de relaciones que van más allá que las puramente lógicas u partitivas y para, además, explicar los intercambios terminológicos incesantes entre diferentes áreas, así como las interdisciplinas, cada vez más frecuentes en el conocimiento actual.

De ahí se derivan algunos de los cambios que va a presentar también la definición, ya que en función del producto establecido podemos necesitar definiciones diferentes (más terminológicas, unas veces; más enciclopédicas, otras veces).

Finalmente, principios como la biunivocidad, con la consiguiente negación sistemática de la existencia de la polisemia y de la sinonímia, deberán ser críticamente replanteados para el trabajo terminológico de manera que, por ejemplo, deberemos tener en cuenta cuales de los diferentes tipos de variación nos interesa recoger, cómo darle su espacio y como representarla.

Por otro lado, las consecuencias metodológicas son evidentes también en cuanto a los posibles productos terminográficos, ya que admitiendo una diversidad de finalidades, de necesidades a cubrir, de destinatarios, etc., tendremos que admitir también una nueva diversidad de productos.

Las informaciones recogidas sobre un término van a tener que adecuarse al tipo de producto previsto, y en función de ello cabrá preguntarse, por ejemplo, qué tipo de fuentes se seleccionan, si es preferible una definición, un contexto de uso o ambas informaciones, si són necesarios campos para recoger la variación (denominativa i/o conceptual) y clasificarla, si són de utilidad informaciones gramaticales sobre colocaciones, si es interesante recoger fraseologías, categorías gramaticales menores, etc.

En cuanto al proceso terminográfico, válido como esquema general de trabajo, es importante proceder a su modernización, a su adecuación a las nuevas tecnologías y herramientas de que se dispone actualmente.

En definitiva, el método de trabajo deberá dejar de ser único y pasar a ser flexible para adecuarse a la diversidad que representan las unidades terminológicas en el marco del conocimiento especializado, teniendo en cuenta la diversidad de la comunicación, la diversidad de los usos linguísticos, el dinamismo de los conceptos, la circularidad del conocimiento, el uso de términos en el discurso y tambien las posibilidades de nuevas tecnologías.

\section{BIBLIOGRAFÍA}

ARNZT, Reiner; PICHT, Heribert. Einführung in die Terminologiearbeit. Hildesheim: Georg Olms, 1989. [Traducción castellana: Introducción a la terminología. Madrid: Fundación Germán Sánchez Ruipérez, 1995].

CABRÉ, María Teresa. La terminologia: la teoria, els mètodes, les aplicacions. Barcelona: Empúries, 1992.

CABRÉ, María Teresa. On diversity and terminology. Terminology, Amsterdam, v. 2, n. 1,1995.

CABRÉ, María Teresa. La terminología: representación y comunicación. Elementos para una teoría de base comunicativa y otros artículos. Barcelona: Institut Universitari de Linguiística Aplicada, Universitat Pompeu Fabra, 1999.

CABRÉ, María Teresa; ROJO, Arcadio. Specialized Knowledge Representation: Towards a New Hypertextual / Multimedia Proposal. In: GALINSKI, Christian; SCHMITZ, Klaus-Dirk. (ed). TKE'96 Terminology and Knowledge Engineering. Frankfurt: Indeks Verlag, 1996.

CIAPUSCIO, Guiomar. El término en los textos: una propuesta integradora para el análisis de la variación conceptual. Ponencia presentada en el VI Simposio de Riterm. L'Havana, nov. 1998.

CONDAMINES, Anne. Terminologie et représentation des connaissances. In: La Banque des Mots, Paris, numéro spécial/6, 1994.

GAMBIER, Yves. Travail et vocabulaires specialisés: prolegomènes à une socioterminologie.In: Meta, Montréal, v. 36, n. 1, 1991.

Organon, Porto Alegre, $\mathrm{n}^{\circ}$ 26, 1998 
GAUDIN, François. Terminology et travail scientifique: mouvement des signes, mouvement des connaissances. In: Cahiers de linguistique sociale, Rouen, núm. 18, 1991.

SLODZIAN, Monique. Comment revisiter la doctrine terminologique aujourd'hui? In: La Banque des Mots, Paris, numéro spécial/ 7, 1995.

TEMMERMAN, Rita. Questioning the univocity ideal. The difference between socio-cognitive Terminology and traditional Terminology. In: Hermes. Journal of Linguistics, núm. 18, p. 51-91, 1997. 